\title{
Extracranial radiosurgery with volumetric modulated arc therapy: Feasibility evaluation of a phase I trial
}

\author{
FRANCESCO DEODATO $^{1}$, SAVINO CILLA ${ }^{2}$, GABRIELLA MACCHIA ${ }^{1}$, LUCIANA CARAVATTA ${ }^{1}$, \\ SAMANTHA MIGNOGNA ${ }^{3}$, MARIANGELA MASSACCESI ${ }^{1}$, VINCENZO PICARDI ${ }^{1}$, CINZIA DIGESU ${ }^{1}$, \\ GIUSEPPINA SALLUSTIO ${ }^{4}$, PIERLUIGI BONOMO ${ }^{3}$, ANGELO PIERMATTEI $^{5}$, GABRIELLA FERRANDINA $^{6}$, \\ GIOVANNI SCAMBIA $^{7}$, VINCENZO VALENTINI ${ }^{8}$, NUMA CELLINI $^{8}$ and ALESSIO G MORGANTI ${ }^{1,3,8}$ \\ Units of ${ }^{1}$ Radiotherapy, ${ }^{2}$ Medical Physics, ${ }^{3}$ Palliative Therapies, ${ }^{4}$ Radiology and ${ }^{6}$ Gynaecologic Oncology, \\ Fondazione di Ricerca e Cura “Giovanni Paolo II”, Università Cattolica del S. Cuore; \\ Departments of ${ }^{5}$ Medical Physics, ${ }^{7}$ Gynaecologic Oncology and ${ }^{8}$ Radiotherapy, Università Cattolica del S. Cuore, \\ I-86100 Campobasso, Italy
}

Received September 13, 2012; Accepted December 3, 2012

DOI: $10.3892 / 01.2013 .1276$

\begin{abstract}
The aim of this study was to report early clinical experience in stereotactic body radiosurgery (SBRS) delivered using volumetric intensity modulated arc therapy (VMAT) in patients with primary or metastatic tumors in various extracranial body sites. Each enrolled subject was included in a different phase I study arm, depending on the tumor site and the disease stage (lung, liver, bone, metastatic), and sequentially assigned to a particular dose level. Technical feasibility and dosimetric results were investigated. The acute toxicity, tumor response and early local control were also studied. In total, 25 lesions in 20 consecutive patients (male/female, 11/9; median age, 67 years; age range, $47-86$ years) were treated. Of these 25 lesions, 4 were primary or metastatic lung tumors, 6 were liver metastases, 8 were bone metastases and 7 were nodal metastases. The dose-volume constraints for organs at risk (OARs) were observed in 19 patients using a single-arc technique. Only in one patient were two arcs required. The treatment was performed without interruption or any other technical issues. The prescribed dose ranged from 12-26 Gy to the planning target volume (PTV). Delivery time ranged from $4 \mathrm{~min}$ to $9 \mathrm{~min}$ and $13 \mathrm{sec}$ (median, $6 \mathrm{~min}$ and $6 \mathrm{sec}$ ). No incidence of grade 2-4 acute toxicity was recorded. The overall response rate was $48 \%$ (95\% confidence interval (CI), 24.2-70.2) based on computed tomography (CT)/magnetic resonance imaging (MRI) and 89\% (95\% CI, 58.6-98.7) based
\end{abstract}

Correspondence to: Dr Gabriella Macchia, Radiotherapy Unit, Fondazione di Ricerca e Cura "Giovanni Paolo II", Università Cattolica del S. Cuore, Largo A. Gemelli 1, I-86100 Campobasso, Italy

E-mail: gmacchia@rm.unicatt.it

Key words: extracranial radiosurgery, stereotactic body radiosurgery, volumetric modulated arc therapy, volumetric intensity modulated arc therapy, feasibility, phase I on the positron emission tomography (PET) scan. SBRS delivered by means of VMAT allowed the required target coverage to be achieved while remaining within the normal tissue dose-volume constraints in the 20 consecutive patients. VMAT-SBRS resulted in adequate technical feasibility; the maximum tolerable dose has not yet been reached in any study arm.

\section{Introduction}

The term stereotactic body radiosurgery (SBRS) implies the delivery of a focused single dose of radiation therapy (1). This technique has been used in the treatment of various types of cancer in different anatomic sites, including primary or metastatic lung tumors (2-4), primary or secondary liver tumors (5-7), pancreatic tumors (8), gynecological cancer recurrences (9) and bone metastases (10).

With the delivery of a very high dose single fraction of radiation therapy, SBRS requires steep dose gradients, usually obtained by dynamic techniques or non-coplanar fixed fields. SBRS also requires high precision in the treatment delivery process. Therefore, it requires a short fraction length to reduce the risk of intra-fraction set-up deviations or organ motion.

Volumetric modulated arc therapy (VMAT) is a novel radiotherapy technique. VMAT differs both from standard intensity-modulated radiation therapy (IMRT) and threedimensional conformal radiotherapy (3D-CRT), which operate in static conditions, and is characterized by dose delivery by dynamic arcs (11). During VMAT, the delivery of radiation occurs with a rotational movement of the linear accelerator (LINAC) gantry while a continuous variation of the beam's profile and intensity is obtained. VMAT requires a sophisticated technique for complex treatment planning. As VMAT has evolved from IMAT, VMAT has the advantage of high-dose conformity and improved sparing of healthy tissues. Therefore, VMAT may be theoretically useful for dose escalation and improved tumor control probability. In addition, the duration of dose delivery is very short, allowing the advantages 
of IMRT (high conformity index) to be combined in a reduced treatment time. The consequences are represented by a higher operating efficiency of each treatment unit, enhanced patient comfort and reduced risk of intrafraction deviations both in terms of set-up errors or organ motion.

For these reasons, VMAT is a potentially ideal technique for SBRS. However, it is not yet clear whether administration of very high doses in single fraction delivery with such a complex technique is possible. Additionally, the true capacity of VMAT to respect dose-volume constraints even in the case of high doses per fraction is uncertain. To the best of our knowledge, no data on VMAT-SBRS have been published.

Based on this background, a feasibility study regarding SBRS based on the VMAT technique (DESTROY-2 protocol) has been planned. The purpose of this analysis is to report the preliminary results of this study.

\section{Materials and methods}

Study characteristics. This trial was conceived as a prospective dose escalation study. All patients consecutively observed at our Radiotherapy Unit (Catholic University, Campobasso, Italy) and matching the inclusion criteria were enrolled. The trial was approved by the Catholic University Institutional Review Board. A preliminary evaluation of technical feasibility was planned following the enrollment of the first 20 patients. Written informed patient consent was obtained from the patients.

Study objectives. The primary study end point was the definition of maximum tolerated dose (MTD) of SBRS with VMAT. The secondary objectives of the study were: i) feasibility evaluation in terms of dose-volume constraints; ii) analysis of the correlation between dosimetric and toxicity data; iii) analysis of the clinical response and iv) evaluation of local control.

Radiosurgery dose escalation. Each enrolled subject was included in a study arm according to the tumor site and disease stage, as demonstrated in Table I. Patients were sequentially assigned to a specific dose level as detailed in Table II. VMAT dose escalation was based primarily on the acute and subacute toxicity, as late toxicity is capable of occurring months or years later. Acute-subacute toxicities were defined as those that occurred within 6 months of receiving treatment. Toxicities registered $\geq 6$ months post-radiation were defined as late toxicities. Dose-limiting toxicities (DLTs) were defined as any treatment-related non hematological adverse effects rated as $\geq$ grade 3 or any hematological toxicity rated as $\geq$ grade 4 , by the National Cancer Institute Common Terminology Criteria for Adverse Events v.4.03 (12). If the DLT was not observed in the three patients at a given dose level, the trial proceeded to the next dose level, provided that 6 months of follow-up had occurred following the VMAT for the third patient of the cohort. If a DLT occurred in one of the three patients at a given dose level, treatment of up to three additional patients at this dose level was required. If the DLT occurred in more than one patient of the three patients' cohort, dose escalation was halted, and the dose level below that was considered to be the MTD. If a DLT occurred in two or more patients of the
Table I. Inclusion criteria.

\begin{tabular}{ll}
\hline Study arm & \multicolumn{1}{c}{ Criteria } \\
\hline Lung & Primary or secondary lung tumors \\
& Number of lesions: $1-5$ \\
& Largest diameter $<5 \mathrm{~cm}$ \\
& Surgical treatment not indicated \\
& No prior RT at the same site \\
& No chemotherapy 14 days before and after SBRS \\
& Absence of bronchopulmonary \\
& Infections in active phase \\
& Primary or secondary liver tumors \\
& Number of lesions $\leq 3$ \\
& (four if two lesions $<3$ cm and close together) \\
Liver & Largest diameter $<6$ cm $(5$ cm for 1 lesion, \\
& 4 cm for 2 lesions, and 3 cm for 3 lesions $)$ \\
& Distance $>6$ mm from the gastrointestinal tract \\
& Surgical treatment not indicated \\
& No previous RT to the liver \\
& No chemotherapy 14 days before and after SBRS \\
Absence of active liver infections & Bone metastases \\
Number of lesions: $1-5$ \\
Largest diameter of the single lesion $<6 \mathrm{~cm}$ \\
Advanced primary tumor or \\
local recurrence or distant metastasis \\
Surgical treatment not indicated \\
Excluded from other arms of the study \\
\end{tabular}

RT, radiotherapy; SBRS, stereotactic body radiosurgery.

Table II. Dose levels (Gy) planned and reached (underlined) in the different arms of the study.

\begin{tabular}{lcccc}
\hline Level & Lung & Liver & Bone & Advanced \\
\hline 1 & $\underline{26}$ & $\underline{26}$ & 12 & 16 \\
2 & 28 & 28 & 14 & $\underline{18}$ \\
3 & 30 & 30 & $\underline{16}$ & 20 \\
4 & 32 & 32 & 18 & 22 \\
5 & 34 & & 20 & 24 \\
6 & & & 22 & \\
7 & & & 24 & \\
\hline
\end{tabular}

expanded six-patient cohort, dose escalation was terminated, and the dose level below that was considered to be the MTD. If a DLT occurred in less than two patients of the expanded six-patient cohort, the trial proceeded to the subsequent dose level. Different total VMAT doses were selected on initiation of the study as the highest dose levels to be evaluated, and were dependent on the study arm. Late toxicities were continuously monitored regardless of whether patients had documented disease progression. 


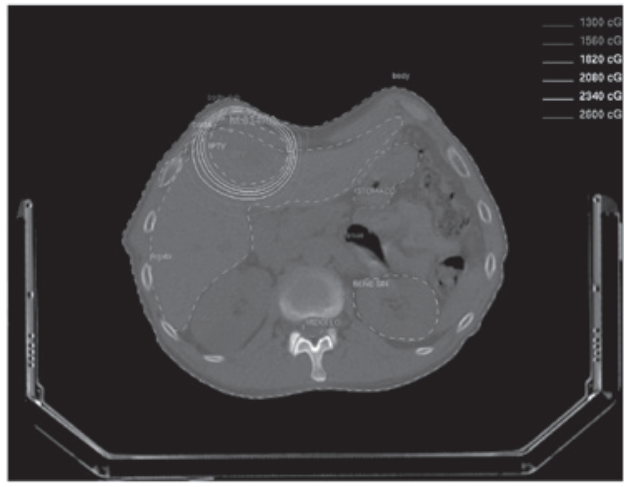

Figure 1. Example of dose distribution.

Inclusion criteria. The following inclusion criteria were used: histological diagnosis of solid tumor (with the exception of germinal tumors) with the site and tumor stage as demonstrated in Table I; age, >18 years; ECOG performance status, 0-3; adequate bone marrow function, which included neutrophil count, $>1500 \mu \mathrm{l}$; platelets, $>100,000 / \mathrm{ml}$; hemoglobin, $>9 \mathrm{~g} / \mathrm{dl}$. Additionally, for patients receiving irradiation to the kidney (lumbar/abdominal area) the inclusion criterion was creatinine, $<1.8 \mathrm{mg} / \mathrm{dl}$; while the criteria for patients receiving irradiation to the liver were total bilirubin, $<3 \mathrm{mg} / \mathrm{dl}$; lactate dehydrogenase, $<3$-fold the normal value; aspartate aminotransferase, $<3$-fold the normal value; alanine aminotransferase, $<3$-fold the normal value and alkaline phosphatase, $<3$-fold the normal value. Previous treatment with surgery and/or chemotherapy and/or radiotherapy was permitted.

Exclusion criteria. The following exclusion criteria were employed: ECOG, $>3$; the presence of medical conditions which contraindicate radiation therapy, such as connective system disorders, severe uncompensated heart disease (in case of heart irradiation), acute diverticulitis, ulcerative colitis and pelvic inflammatory disease (in case of irradiation of the pelvis); comorbidities that in the opinion of the referring physician may constitute a risk to clinical trial participation.

End points and statistical analysis. Toxicity was evaluated by the Common Toxicity Criteria for Adverse Events (CTCAE) scale, version 4.03 (12). The presence of focal liver reaction was evaluated as outlined by Herfarth et al (13). The survival curves were calculated with the Kaplan-Meier method (14). Statistical analysis was performed using SYSTAT software, version 11.0 (SPSS, Inc.; Chicago, IL, USA).

Patient set-up. Patient immobilization was performed with a stereotactic body frame (SBF; Elekta; Crawley, UK), which is an immobilization device used to define a stereotactic system of coordinates for the target position as opposed to anatomical landmarks such as bony structures or skin markers. This device was described in detail by Lax et al (15) and clinical results have been published by Blomgren et al (16). The SBF is a U-shaped rigid plastic frame, within which different sized vacuum pillows allow a reproducible immobilization for the repositioning of each patient. Patient repositioning is supported by a laser system directly attached to the body frame at defined longitudinal positions. Alignment of the stereotactic coordinate system of the SBF to the isocenter of the computed tomography (CT) machine or the treatment unit is performed by a stereotactic arc with scales in the anterior-posterior and lateral directions. The longitudinal stereotactic coordinate is found on a scale along the body frame sidewalls and is simply read on each CT-slice using a system of straight and oblique copper pieces, which function as fiducials. Moreover, to reduce the respiration mobility of targets close to the diaphragm, a compressor, attached to the SBF by a rigid arc, may be mechanically pressed into the patient's epigastrium to decrease the respiration motion.

CT simulation. To evaluate the reproducibility of the set-up, three CT scan evaluations were performed on three different days, with the aim of verifying that the set-up deviation was $<3 \mathrm{~mm}$. In order to evaluate the organ motion produced by the respiratory movements, target displacement was measured. During free breathing, 30 axial CT scans were performed on the same slice. In the case of a displacement $>5 \mathrm{~mm}$, the abdominal compressor of the SBF was applied and the CT scan for organ motion assessment was repeated. The final CT simulation, for the acquisition of axial images necessary for stereotactic localization and plan calculations, was produced with a spiral technique. Subsequently, 3-mm scans were acquired with a $3-\mathrm{mm}$ interval between scans in the target region. For the remainder of the SBF, 10-mm slices were acquired and the interval between scans was $10 \mathrm{~mm}$. In treating abdominal or pelvic targets, patients received $2 \mathrm{cc}$ of oral Gastrografin, diluted in 0.51 of water $30 \mathrm{~min}$ prior to CT scan acquisition. In case of mediastinal, abdominal or pelvic target volumes, intravenous infusion of an iodinated contrast medium was also used.

Volumes of interest. The clinical target volume (CTV) was defined as the gross tumor volume (GTV) in case of metastases and primary lung tumors. A 5-mm margin was added to the GTV to define the CTV in primary tumors of the liver. The planning target volume (PTV) was individually defined for each patient based on the internal margin (IM) and the set-up margin (SM) assessment. The IM was defined based on respiratory excursions in $3 \mathrm{D}$. The $\mathrm{SM}$ was set at $3 \mathrm{~mm}$ according to the ROSEL study (17). The OARs considered included: i) The thorax: the spinal cord, lungs, esophagus, heart, brachial plexus, peripheral nerves, large vessels, trachea and ribs; ii) The abdomen: the spinal cord, liver, stomach, small bowel, colon and kidneys; iii) The pelvis: the sacral plexus, small bowel, colon, rectum, anal canal, bladder, femoral heads and penile bulb.

Prescription. A uniform method for the selection of the prescription isodose surface (IDS) was adopted. According to the ROSEL study (17), for each plan the IDS was selected as the greatest IDS fulfilling the two following criteria: $95 \%$ of the PTV volume reached $100 \%$ of the prescription dose and $99 \%$ of the PTV reached $\geq 90 \%$ of the prescription dose. The aim was to increase the dose heterogeneity so as to intensify the dose within the GTV. The maximum dose within the PTV should not exceed $140 \%$ of the prescribed dose. Careful attention was paid to ensure the maximum dose always remained within the GTV. 
Table III. Dose-volume constraints.

\begin{tabular}{lll}
\hline Organ & Dose $(\mathrm{Gy})$ or volume (\% or cc) & Reference \\
\hline Ribs & $\mathrm{Dmax}=30$ & NCCN v.2.2010 (18) \\
Heart/pericardium & $\mathrm{Dmax}=22$ & NCCN v.2.2010 (18) \\
Skin & $\mathrm{Dmax}=26$ & NCCN v.2.2010 (18) \\
Esophagus & $\mathrm{Dmax}=15.4$ & NCCN v.2.2010 (18) \\
Liver & $\mathrm{V}_{12 \mathrm{~Gy}}<30 \%$ & Herfarth KK, 2001 (5) \\
& $\mathrm{V}_{7 \mathrm{~Gy}}<50 \%$ & NCCN v.2.2010 (18) \\
Great vessels (mediastinum) & $\mathrm{Dmax}=37$ & Herfarth KK, 2001 (5) \\
Bowel (small bowel/colon) & $\mathrm{Dmax}=12$ & NCCN v.2.2010 (18) \\
Spinal cord & $\mathrm{Dmax}=14$ & NCCN v.2.2010 (18) \\
Brachial plexus & $\mathrm{Dmax}=17.5$ & Timmerman RD, 2008 (19) \\
Sacral plexus & $\mathrm{Dmax}=18$ & Timmerman RD, 2008 (19) \\
Lungs & $\mathrm{V}_{7.4 \mathrm{~Gy}}=1000$ cc & \\
Kidneys & $\mathrm{V}_{8.4 \mathrm{~Gy}}=800 \mathrm{cc}($ cortical area) & Timmerman RD, 2008 (19) \\
Stomach & $\mathrm{V}_{10.6 \mathrm{~Gy}}=2 / 3$ volume (ilo) & NCCN v.2.2010 (18) \\
Trachea/large bronchus & $\mathrm{Dmax}=12.4$ & NCCN v.2.2010 (18) \\
\hline
\end{tabular}

NCCN, National Comprehensive Cancer Network.

Treatment planning. VMAT plans were generated using the $\mathrm{ERGO}^{++}$treatment planning system (TPS), version 1.7.3 (Elekta). This is an anatomy-based TPS that supplies a simplified approach to creating VMAT plans, by predefining a series of aperture shapes using Boolean operations in conjunction with the beam's eye view of the target and OARs. In the current study, all plans were generated with a single-arc rotation except for patient number 16, who was treated for two liver lesions and therefore required two arcs. The dose calculation was performed using the pencil beam algorithm with inhomogeneity correction and a dose grid resolution of $2 \mathrm{~mm}$. VMAT plans were exported to the record and verify $(\mathrm{R} \& \mathrm{~V})$ system Mosaiq v. 1.6 (Impac Software; Elekta) by DICOM-RT for later irradiation. Table III lists the dose-volume constraints used $(5,18,19)$.

Quality assurance. Set-up deviation and organ motion assessments were performed as previously described. For quality assurance through treatment planning and delivery, two independent checks (IC1 and 2) were performed by medical and physics staff, as previously described (20).

Supportive therapy. Supportive therapy was prescribed according to the irradiated site. In the case of irradiation of two anatomic sites, such as the chest and the abdomen, supportive care was provided for both sites. In patients receiving irradiation to the chest, prescriptions included betamethasone $0.5 \mathrm{mg}$ orally, 3 times daily for 1 month, followed by a gradual reduction, associated with gastric protection (H2-inhibitors). Patients receiving abdominal irradiation were prescribed metoclopramide $10 \mathrm{mg}$ orally, 3 times a day, for $\leq 1$ week following radiation therapy and rabeprazole $40 \mathrm{mg}$ orally, once daily for 12 months (in case of irradiation of the stomach and/or the duodenum only). In addition, patients receiving irradiation to the upper abdomen were prescribed dexamethasone $12 \mathrm{mg}$ intravenously (IV) $1 \mathrm{fl}$ immediately prior to radiosurgery and $6 \mathrm{~h}$ after treatment, while $3 \mathrm{mg}$ granisetron was administered immediately prior to radiosurgery by IV slow infusion.

Evaluation of response and follow-up. The tumor response assessment was performed 8-12 weeks after treatment. Morphological imaging modalities were employed (CT with contrast medium and/or MRI with or without contrast) in all patients. Using this method, the tumor response was based on the response evaluation criteria in solid tumors (RECIST) criteria (21). If feasible, the response was also assessed with functional imaging, which included $\left({ }^{18} \mathrm{~F}\right)$-fluorodeoxyglucose (FDG)-PET or choline PET for prostate cancer. In this study, the European Organisation for Research and Treatment of Cancer (EORTC) criteria were used (22). Specifically, the PET-based response was assessed according to criteria including progressive metabolic disease (PMD), stable metabolic disease (SMD), a partial metabolic response (PMR) and a complete metabolic response (CMR). PMD involved an increase in the tumor $\left({ }^{18} \mathrm{~F}\right)-\mathrm{FDG}$ standardized uptake value (SUV) of $>25 \%$ within the tumor region defined on the baseline scan, a visible increase in the extent of tumor $\left({ }^{18} \mathrm{~F}\right)-\mathrm{FDG}$ uptake of $>20 \%$ in the longest dimension, or the appearance of novel tumor $\left({ }^{18} \mathrm{~F}\right)-\mathrm{FDG}$ uptake in metastatic lesions. SMD comprised an increase in the tumor $\left({ }^{18} \mathrm{~F}\right)$-FDG SUV of $<25 \%$ or a decrease of $<15 \%$, and no visible increase in the extent of the $\left({ }^{18} \mathrm{~F}\right)$-FDG tumor uptake (i.e., not $>20 \%$ in the longest dimension). A partial metabolic response required a reduction of $>25 \%$ in the tumor $\left({ }^{18} \mathrm{~F}\right)$-FDG SUV. A reduction in the extent of the tumor $\left({ }^{18} \mathrm{~F}\right)-\mathrm{FDG}$ uptake was not a pre-requesite for a PMR, whereas a CMR negated a complete resolution of the $\left({ }^{18} \mathrm{~F}\right)-\mathrm{FDG}$ uptake within the tumor volume, in order that it was indistinguishable from the surrounding normal tissue. The 
Table IV. Follow-up.

\begin{tabular}{ll}
\hline $\begin{array}{l}\text { Study arm } \\
\text { Lung } \\
\text { Liver }\end{array}$ & \multicolumn{1}{c}{ First follow-up } \\
& Subsequent follow-up \\
Bone & Chest CT and PET-CT at 3 months and every 6 months thereafter \\
& Abdominal CT and PET-CT at 3 months and every \\
to evaluate acute toxicity & 6 months thereafter; focal hepatic reaction evaluation \\
Advanced & Bone CT and PET-CT or bone-scan at 3 months \\
& and every 6 months thereafter (anticipated if symptoms) \\
& Body CT and PET-CT at 3 months and every \\
& 6 months thereafter (anticipated if symptoms)
\end{tabular}

SBRS, stereotactic body radiosurgery; CT, computed tomography; PET, positron emission tomography.

A

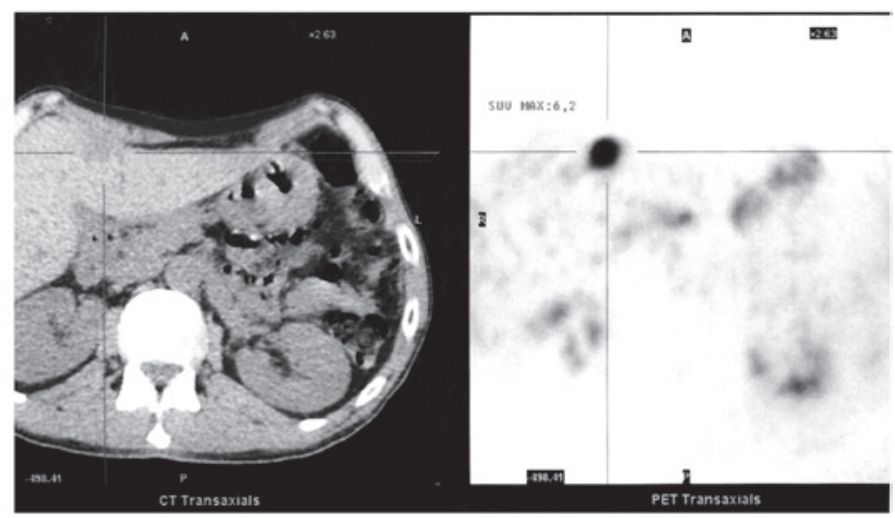

$\mathbf{B}$

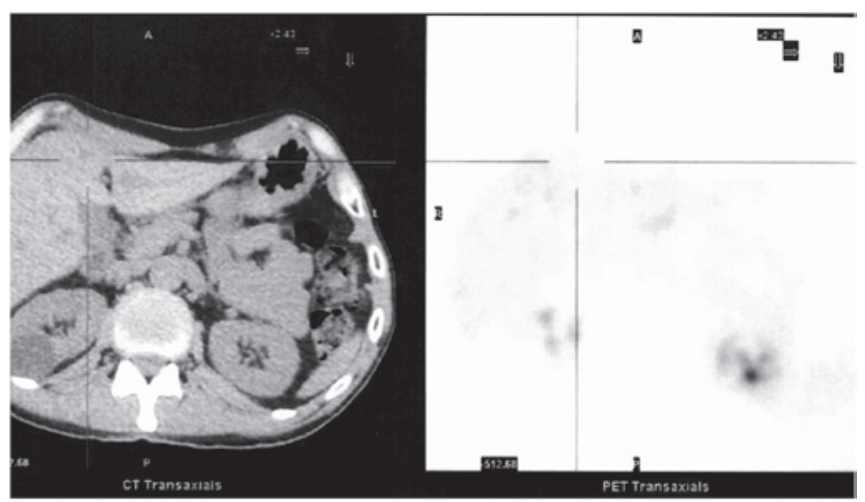

Figure 2. Single liver metastasis: Fluorodeoxyglucose (FDG)-positron emission tomography (PET) before (A) and after (B) radiosurgery (26 Gy).

follow-up was performed according to the scheme detailed in Table IV.

Quality of life (QoL) evaluation. The cancer linear analog scale (CLAS) score was used to evaluate the impact of SBRS on the patient's quality of life (CLAS1), energy level (CLAS2) and ability to undertake daily activities (CLAS3), both prior to and 3-4 weeks after radiotherapy. Patients scored their perceptions of these symptoms by placing a mark on a 100-mm line (23).

\section{Results}

Patient characteristics. The preliminary analysis was based on the first 20 enrolled patients who had a total of 25 lesions
(Table V). The median PTV size was 37.8 cc (range, 0.9-202.4). The prescribed dose ranged from 12-26 Gy to the PTV (Fig.1).

Technical issues. The dose-volume constraints for OARs were observed in all patients using a single-arc technique. Only one patient, who was treated for two liver lesions, required a two-arc technique. To administer the prescribed doses, 1401.9-3246.2 monitor units (median, 2157.75) were employed with a median beam-on time of $6 \mathrm{~min}$ and $6 \mathrm{sec}$ (range, $4 \mathrm{~min}$ and $0 \mathrm{sec}$ to $9 \mathrm{~min}$ and $13 \mathrm{sec}$ ). In all patients, the treatment was performed without interruption or any other technical issues.

Acute toxicity and response. All patients were evaluable for acute toxicity. Twenty per cent of patients experienced grade 1 acute toxicity. No patients demonstrated acute toxicity 


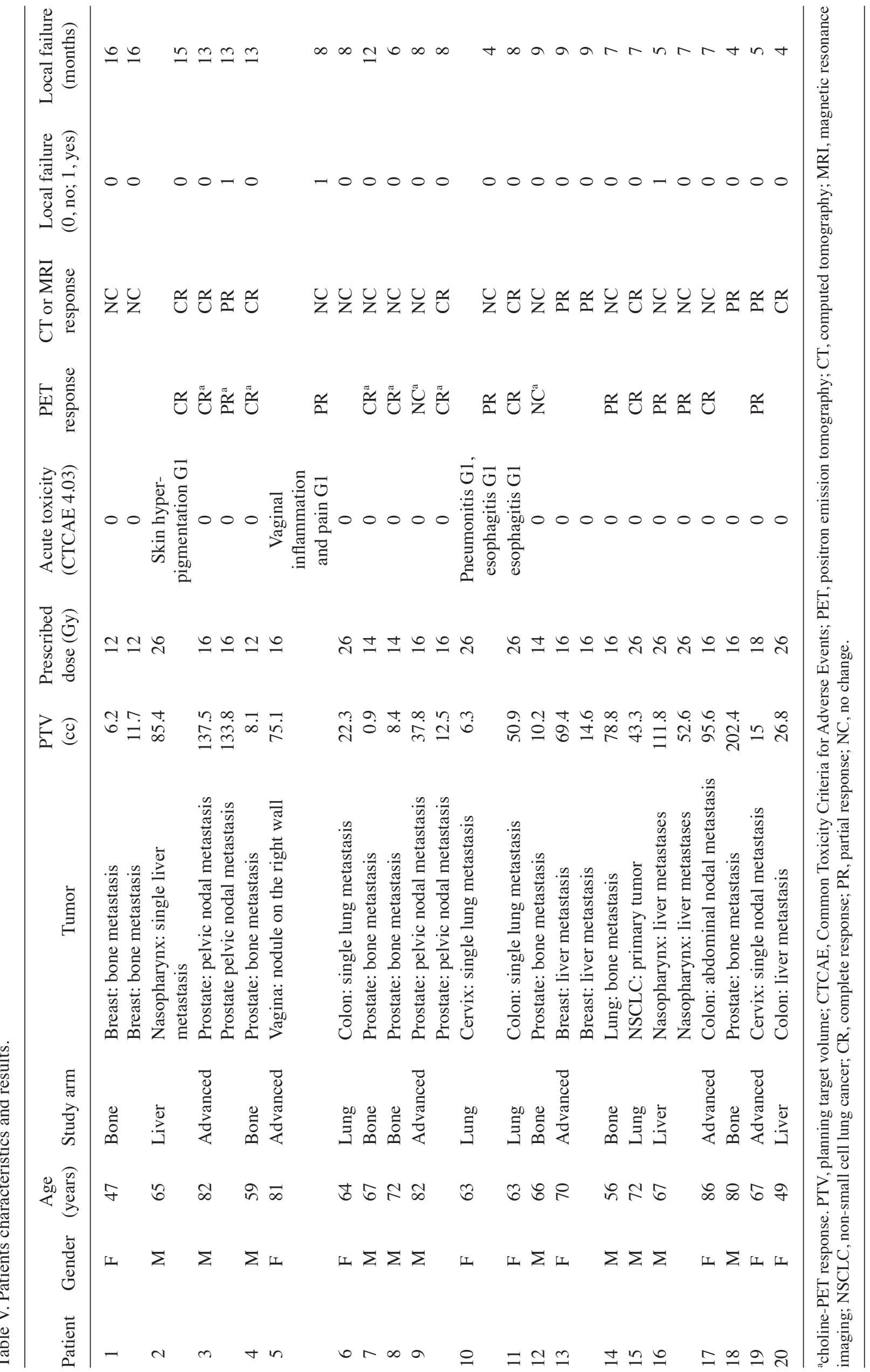


$>$ grade 1 . Twenty five lesions were evaluable for clinical response by morphological imaging. In the irradiated site, the tumor responses included 7 lesions with a complete response (CR; 28\%), 5 with a partial response (PR; 20\%) and 13 with stable disease (SD; 52\%). Moreover, 18 lesions were evaluable for a clinical response by functional imaging as follows: 9 lesions with a CR (50\%), 7 with a PR (39\%) and 2 with SD $(11 \%)$ (Fig. 2). No difference in the CLAS score was observed prior to SBRS compared with at the first follow-up (data not shown).

Late toxicity and outcome. With a median follow-up time of 12 months (range, 8-20), no patients presented with late toxicity. Overall, 3 patients experienced local disease progression. One-year actuarial progression-free survival in the irradiated site was $88 \%$, while 13 patients $(65 \%)$ demonstrated progressive disease in sites different from the irradiated one.

\section{Discussion}

We describe our initial experience with radiosurgery by VMAT. Large radiation doses were delivered to the 20 patients in this study, and the constraints of the OARs were observed and a simple single-arc technique was implemented (in 19/20 patients) in $<10 \mathrm{~min}$. Acute toxicity was exclusively grade 1 (CTCAE 4.03). Considering the 25 lesions, a morphological response rate of $48 \%(95 \% \mathrm{CI}, 24.2-70.2)$ and a functional response rate of $89 \%$ (95\% CI, 58.6-98.7) were demonstrated.

There are few studies in the literature regarding the use of stereotactic VMAT. The majority of these are dosimetric studies concerned with spine (24,25), lung (24,26-28), brain $(29,30)$ and adrenal metastases (31). All of these studies have demonstrated an increased efficiency of VMAT in terms of treatment time, with respect to 3D-conformal or IMRT techniques. A number of these have also described improved conformity compared with $3 \mathrm{D}$ techniques (26-28) and a similar $(30)$ or higher $(27,28)$ conformity compared with standard IMRT techniques. Clinical studies are less numerous and are concerned with the spine (32-34), arteriovenous malformations (35) and abdominal targets (36). These preliminary studies have mainly documented the technical feasibility of stereotactic VMAT, and all the authors have employed this technique in fractionated treatments. To the best of our knowledge, the present series represents the first clinical study on radiosurgery using VMAT.

In terms of feasibility, we stress that the dose-volume constraints were met in all patients in the current study. The use of relatively small doses, in this first phase of the study, likely facilitated this result. In addition, the use of high doses was tolerated at least in terms of acute toxicity. Moreover, the analysis of QoL- and fatigue-related indicators prior to and following radiosurgery demonstrated that SBRS was not associated with any detrimental effects. The low number of patients and the short follow-up time mean that is is not possible to assess the local control and late toxicity. However, the high index of an immediate response, particularly if assessed with functional imaging, and the absence of relevant toxicity should be noted.

From a practical perspective, introducing VMAT for radiosurgery resulted in a marked reduction in the treatment time.
In our previous experience with stereotactic radiation therapy based on non-coplanar fixed fields, a time of 45 min was reserved for each treatment. In the present study concerning VMAT, a machine time of only $20 \mathrm{~min}$ per treatment was reserved. Considering the promising results in terms of the feasibility and the preliminary clinical results, the study should continue with the recruitment of additional patients to the subsequent dose levels.

\section{References}

1. Potters L, Steinberg M, Rose C, et al: American Society for Therapeutic Radiology and Oncology and American College of Radiology practice guidelines for the performance of stereotactic body radiation therapy. Int J Radiat Oncol Biol Phys 60: 1026-1032, 2004.

2. Nagata Y, Matsuo Y, Takayama K, et al: Current status of stereotactic radiotherapy for lung cancer. Int J Clin Oncol 12: 3-7, 2007.

3. Nagata Y, Negoro Y, Aoki T, et al: Clinical outcomes of 3D conformal hypofractionated single high-dose radiotherapy for one or two lung tumors using a stereotactic body frame. Int J Radiat Oncol Biol Phys 52: 1041-1046, 2002.

4. Nakagawa K, Aoki Y, Tago M, Terahara A, Ohtomo K: Megavoltage CT-assisted stereotactic radiosurgery for thoracic tumors: original reseach in the treatment of thoracic neoplasms. Int J Radiat Oncol Biol Phys 48: 449-457, 2000.

5. Herfarth KK, Debus J, Lohr F, et al: Stereotactic single-dose radiation therapy of liver tumors: results of a phase I/II trial. J Clin Oncol 19: 164-170, 2001.

6. Wulf J, Guckenberger M, Haedinger U, et al: Stereotactic radiotherapy of primary liver cancer and hepatic metastases. Acta Oncol 45: 838-847, 2006.

7. Kavanagh BD, McGarry RC and Timmerman RD: Extracranial radiosurgery (stereotactic body radiation therapy) for oligometastases. Semin Radiat Oncol 16: 77-84, 2006.

8. Koong AC, Christofferson E, Le QT, et al: Phase II study to assess the efficacy of conventionally fractionated radiotherapy followed by a stereotactic radiosurgery boost in patients with locally advanced pancreatic cancer. Int J Radiat Oncol Biol Phys 63: 320-323, 2005.

9. Deodato F, Macchia G, Grimaldi L, et al: Stereotactic radiotherapy in recurrent gynecological cancer: a case series. Oncol Rep 22:415-419, 2009.

10. Gerszten PC, Ozhasoglu C, Burton SA, et al: CyberKnife frameless stereotactic radiosurgery for spinal lesions: clinical experience in 125 cases. Neurosurgery 55: 89-98, 2004.

11. Song Y, Zhang P, Wang P, et al: The development of a novel radiation treatment modality-volumetric modulated arc therapy. Conf Proc IEEE Eng Med Biol Soc 2009: 3401-3404, 2009.

12. National Cancer Institute. Common Terminology Criteria for Adverse Events v.4.03 (CTCAE v.4.03). Available at: http://ctep. cancer.gov/protocolDevelopment/electronic_applications/ctc.htm. Accessed February 22, 2012.

13. Herfarth KK, Hof H, Bahner ML, et al: Assessment of focal liver reaction by multiphasic CT after stereotactic single-dose radiotherapy of liver tumors. Int J Radiat Oncol Biol Phys 57: 444-451, 2003.

14. Kaplan EL and Meier P: Non-parametric estimation from incomplete observations. J Am Statist Assoc 53: 457-481, 1985.

15. Lax I, Blomgren H, Näslund I and Svanström R: Stereotactic radiotherapy of malignancies in the abdomen. Methodological aspects. Acta Oncol 33: 677-683, 1994.

16. Blomgren H, Lax I, Näslund I and Svanström R: Stereotactic high dose fraction radiation therapy of extracranial tumors using an accelerator. Clinical experience of the first thirty-one patients. Acta Oncol 34: 861-870, 1995.

17. Hurkmans CW, Cuijpers JP, Lagerwaard FJ, et al: Recommendations for implementing stereotactic radiotherapy in peripheral stage IA non-small cell lung cancer: report from the Quality Assurance Working Party of the randomised phase III ROSEL study. Radiat Oncol 4: 1, 2009.

18. National Comprehensive Cancer Network (NCCN). Practice Guidelines in Oncology v.2.2010-Non Small Cell Lung Cancer.

19. Timmerman RD: An overview of hypofractionation and introduction to this issue of seminars in radiation oncology. Semin Radiat Oncol 18: 215-222, 2008. 
20. Morganti AG, Deodato F, Zizzari S, et al: Complexity index (COMIX) and not type of treatment predicts undetected errors in radiotherapy planning and delivery. Radiother Oncol 89: 320-329, 2008

21. Therasse P, Arbuck SG, Eisenhauer EA, et al: New guidelines to evaluate the response to treatment in solid tumors. J Natl Cancer Inst 92: 205-216, 2000.

22. Young H, Baum R, Cremerius U, et al: Measurement of clinical and subclinical response using $[18 \mathrm{~F}]$-fluorodeoxiglucose and Positron Emission Tomography: review and 1999 EORTC recommendations. European Organisation for Research and Treatment of Cancer (EORTC) PET Study Group. Eur J Cancer 35: 1773-1782, 1999.

23. Sutherland HJ, Walker P and Till JE: The development of a method for determining oncology patients' emotional distress using linear analogue scales. Cancer Nurs 11: 303-308, 1988.

24. Matuszak MM, Yan D, Grills I and Martinez A: Clinical applications of volumetric modulated arc therapy. Int J Radiat Onco Biol Phys 77: 608-616, 2010.

25. Kuijper IT, Dahele M, Senan S and Verbakel WF: Volumetric modulated arc therapy versus conventional intensity modulated radiation therapy for stereotactic spine radiotherapy: a planning study and early clinical data. Radiother Oncol 94: 224-228, 2010.

26. McGrath SD, Matuszak MM, Yan D, Kestin LL, Martinez AA and Grills IS: Volumetric modulated arc therapy for delivery of hypofractionated stereotactic lung radiotherapy: A dosimetric and treatment efficiency analysis. Radiother Oncol 95: 153-157, 2010.

27. Holt A, van Vliet-Vroegindeweij C, Mans A, Belderbos JS and Damen EM: Volumetric-modulated arc therapy for stereotactic body radiotherapy of lung tumors: a comparison with intensitymodulated radiotherapy techniques. Int J Radiat Oncol Biol Phys 81: 1560-1567, 2011

28. Ong CL, Verbakel WF, Cuijpers JP, Slotman BJ, Lagerwaard FJ and Senan S: Stereotactic radiotherapy for peripheral lung tumors: a comparison of volumetric modulated arc therapy with 3 other delivery techniques. Radiother Oncol 97: 437-442, 2010.
29. Ma Y, Li M, Yin Y, et al: Hypofractionated stereotactic radiotherapy for brain metastases: a dosimetric and treatment efficiency comparison between volumetric modulated arc therapy and intensity modulated radiotherapy. Technol Cancer Res Treat 9: 499-507, 2010.

30. Mayo CS, Ding L, Addesa A, Kadish S, Fitzgerald TJ and Moser R: Initial experience with volumetric IMRT (RapidArc) for intracranial stereotactic radiosurgery. Int J Radiat Oncol Biol Phys 78: 1457-1466, 2010.

31. Scorsetti M, Mancosu P, Navarria P, et al: Stereotactic body radiation therapy (SBRT) for adrenal metastases: a feasibility study of advanced techniques with modulated photons and protons. Strahlenther Onkol 187: 238-244, 2011.

32. Palma DA, van Sörnsen de Koste J, Verbakel WF, Vincent A and Senan S: Lung Density Changes After Stereotactic Radiotherapy: A Quantitative Analysis in 50 Patients. Int J Radiat Oncol Biol Phys 81: 974-978, 2011.

33. Palma DA, Senan S, Haasbeek CJ, Verbakel WF, Vincent A and Lagerwaard F: Radiological and clinical pneumonitis after stereotactic lung radiotherapy: a matched analysis of threedimensional conformal and volumetric-modulated arc therapy techniques. Int J Radiat Oncol Biol Phys 80: 506-513, 2011.

34. Ong CL, Palma D, Verbakel WF, Slotman BJ and Senan S: Treatment of large stage I-II lung tumors using stereotactic body radiotherapy (SBRT): planning considerations and early toxicity. Radiother Oncol 97: 431-436, 2010.

35. Subramanian S, Srinivas C, Ramalingam K, et al: Volumetric modulated arc-based hypofractionated stereotactic radiotherapy for the treatment of selected intracranial arteriovenous malformations: dosimetric report and early clinical experience. Int J Radiat Oncol Biol Phys 82: 1278-1284, 2012.

36. Scorsetti M, Bignardi M, Alongi F, et al: Stereotactic body radiation therapy for abdominal targets using volumetric intensity modulated arc therapy with RapidArc: feasibility and clinical preliminary results. Acta Oncol 50: 528-538, 2011. 